\title{
LINEAR QUANTUM MEASUREMENTS
}

\author{
D.V. AVERIN \\ Department of Physics and Astronomy, \\ Stony Brook University, SUNY \\ Stony Brook, New York 11794-3800, U.S.A.
}

\begin{abstract}
.
Linear response theory describes quantum measurement with an arbitrary detector weakly coupled to a measured system. This description produces generic quantitative relation characterizing the detector that is analogous to the fluctuation-dissipation theorem for equilibrium systems. The detector characteristic obtained in this way shows how efficient the trade-off is between the back-action dephasing and information acquisition by the detector.
\end{abstract}

\section{Introduction}

If one puts aside philosophical questions created by the perceived counterintuitive features of the quantum mechanics that are frequently discussed in the literature on the quantum measurement problem (an entry point to this literature is provided, e.g., by the collections of papers $[1,2,3]$ or monographs $[4,5])$ it is easy to see that the physics of quantum measurements is fairly well understood by now at least on a qualitative level. The process of quantum measurement is dynamic interaction between a microscopic quantum system and a macroscopic detector that establishes correlations between the states of these systems. Although it is impossible to give a universal definition of "macroscopic" in this context, a reasonable working definition is that the macroscopic detector is a system with negligible quantum fluctuations. Such systems are quite abundant in nature and can be described quantitatively within the framework of quantum mechanics.

Since the description of quantum measurement process as an interaction between microscopic and macroscopic systems is quite broad, it is of interest to see whether there are any universal quantitative features of this process 
that are independent of specific physical realization of the detectors and the measured system. The purpose of this work is to show that one such universal characteristics of quantum measurements can be obtained from the linear response theory. This result was first pointed out in [6], and this work gives its more concise and accurate presentation. ${ }^{1}$

An example of the area where a universal description of the quantum measurement process can be particularly useful is mesoscopic quantum dynamics of solid-state, in particular Josephson-junction, qubits. Recent widespread interest in the development of solid-state qubits for quantum information processing brought with it the discussion of a large number of different detectors for measurement of quantum dynamics of these qubits. These detectors include quantum point contacts $[7,8,9,10,11]$, normalmetal $[12,13,14,15]$ and superconducting $[16,17]$ SET transistors operated in different regimes, resonant tunneling structures [18], generic mesoscopic conductors [19], SQUIDs [20]. With such a variety of detectors (the list of references above is by no means complete), a possibility of giving a quantitative description of some detector characteristics independently of its physical realization is obviously very helpful for understanding of the process of quantum measurement of mesoscopic qubits.

\section{Measurement model and basic relations}

As was discussed in the introduction, the most essential feature of the model of the quantum measurement process considered in this work is its universality. The model applies to measurement with an arbitrary detector which has to satisfy only some basic conditions. Schematics of this model is shown in Fig. 1 and includes the measured system and the detector with the Hamiltonians $H_{S}$ and $H_{D}$, respectively. The detector and the system are coupled through an interaction Hamiltonian $H_{\text {int }}$ which almost without any loss of generality can be written as the product of some system "coordinate" $x$ and the detector "force" $f$ :

$$
H_{\text {int }}=x f
$$

The operator $x$ acts as the observable measured by the detector. In principle, one could imagine a situation when a detector couples to some system through a combination of the several product terms like (1), but the detector sensitive to several different dynamic variables of the system would be quite unusual.

\footnotetext{
${ }^{1}$ Note added in the electronic version. After this manuscript was submitted for publication, the author's attention was drawn to Ref. [27] which presents similar development of Ref. [6].
} 


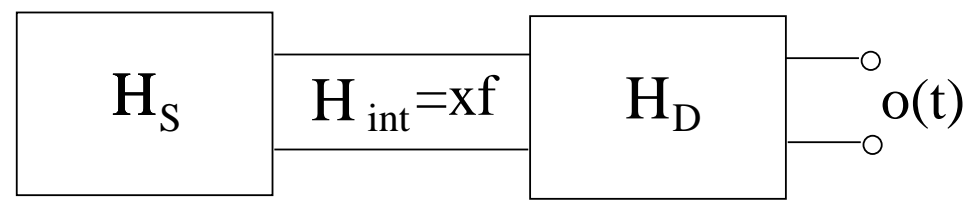

Figure 1. Schematics of the generic quantum measurement process. The detector couples to the measured system via the product of the measured observable $x$ and the detector force $f$ and variation of its state is reflected in the output variable $o$ with essentially classical dynamics.

The general structure of the Hamiltonian of the system:

$$
H=H_{S}+H_{D}+H_{\text {int }},
$$

is similar to the Hamiltonians studied in the discussions of the dissipative quantum dynamics based on the "system+reservoir" models (see, e.g., [21]) if the detector is identified with the reservoir. This analogy is not accidental, and many features of the dynamics of the measurement process are similar to the dissipative quantum dynamics of open systems. For instance, as will also be discussed below, coupling to the detector leads to the dephasing of the measured system, similar to the reservoir-induced dephasing.

There are, however, important differencies between the two situations. The main difference is that in the case of the dissipative quantum dynamics induced by a reservoir, changes in the reservoir caused by the system of interest are assumed to be unobservable, while the detector as a matter of principle should have an output observable $o(t)$ that provides some information about its state. To represent the complete measurement process, $o(t)$ should behave as a classical variable, i.e. magnitude of quantum fluctuations in it should be much smaller than its classical component. This condition implies that there is another difference between the detector and the dissipative reservoir. The detector should be able to convert weak quantum input signal $x(t)$ into the classical output signal $o(t)$ that is large on the scale of quantum fluctuations. Such a transformation requires amplification and can not be achieved in an equilibrium system, i.e., the detector, in contrast to the reservoir has to be driven out of the equilibrium.

In the case of the dissipative reservoirs, it is well known that the linear response theory is a powerful tool that produces a general quantitative statement, fluctuation-dissipation theorem, about the reservoir-induced relaxation. The theorem is independent of the specific microscopic model of the system. The analogy between the measurement and the environmentinduced relaxation leads naturally to the question of whether some general relations characterizing quantum measurements can be derived from the 
linear response theory. As we will see below, the answer to this question is yes, and the linear response theory applied to the measurement problem enables one to derive generic restriction on the energy sensitivity of a general linear quantum detector. The obtained relation characterizes how close the detector is to being quantum-limited, i.e., how close the detector-induced back-action dephasing in the measured system is to the fundamental limit set by the quantum mechanics. While such a restriction on the energy sensitivity was known before in several specific detector models, linear response theory establishes it for an arbitrary detector weakly coupled to the measured system.

For the linear response theory to be applicable to the measurement set-up shown in Fig. 1, and to provide some meaningful information, the detector has to satisfy certain conditions. Two most important are:

- the detector is weakly coupled to the measured system so that its response to variations in this system is linear,

- the detector is in the stationary state.

Since the detector can not be in equilibrium, the second item on this list generalizes the equilibrium condition of the standard linear-response theory. Both of these conditions are not very restrictive, and are satisfied in many experimental situations.

Linearity of the response is not sufficient to specify the detector properties completely, since the linear response coefficient can have an arbitrary frequency dependence, i.e. one needs to assume some spectral characteristics of the detector. Here we consider the simplest case when the response time of the detector is much shorter that the characteristic time of the dynamics of the measured system. This means that the response coefficient $\lambda$ is constant in the relevant frequency range. Although this assumption is less fundamental that the previous two, and in principle can be avoided, it simplifies the discussion considerably.

Quantitatively, linearity of the detector response means that dynamics of the detector operators can be treated in the lowest order of the perturbation theory in the detector-system coupling $H_{\text {int }}$. Expanding the evolution operator $U(t)=e^{-i H t / \hbar}$ with the total Hamiltonian (2) upto the first order in $H_{\text {int }}$ we see that the detector output $o(t)$ can be written as:

$$
o(t)=q(t)+\frac{i}{\hbar} \int^{t} d \tau[f(\tau), q(t)] x(\tau) .
$$

The first term $q(t) \equiv e^{i H_{D} t / \hbar} o(0) e^{-i H t / \hbar}$ in this expression can be interpreted as the output noise of the detector that exists even in the absence of the input signal, while the second term represents the detector's linear response to measured system. In zeroth order in coupling, the detector and 
the system are independent, so the averages over their states in eq. (3) can be taken separately. Tracing out the detector variables in (3) we obtain the regular expression for the linear response, extended to the situation when the "driving force" $x(t)$ is a quantum operator. With the adopted assumption of instantaneous detector response:

$$
\frac{i}{\hbar}\langle[f(t), q(t+\tau)]\rangle_{D}=\lambda \delta(\tau-0)
$$

eq. (3) reduces to:

$$
\langle o(t)\rangle_{D}=\lambda x(t)
$$

The infinitesimal shift in the argument of the $\delta$-function in (4) represents small but finite response time of the detector, and is needed to resolve the ambiguity in eq. (3). In equation (4), we used the assumption (introduced above) that the detector is in the stationary state and the $q-f$ correlator depends therefore only on the difference of the time arguments, and $\langle\ldots\rangle_{D}$ denotes the average of the stationary detector density matrix. Since $q(t)$ represents the output noise, we took $\langle q(t)\rangle_{D}=0$.

Similarly, if the detector produces some non-vanishing average force $\langle f(t)\rangle_{D} \equiv F \neq 0$, it is natural to include it in the system Hamiltonian

$H_{S}$, and one can therefore always assume that $\langle f(t)\rangle_{D}=0$. Dynamics of the coupling force $f$ generated by the detector is characterized then by the correlation function $\langle f f(t)\rangle_{D}$ and physically can be viewed as "backaction" noise that dephases the measured system. Following the assumption of the instantaneous response (4) we also take both the output noise and the back-action noise to be $\delta$-correlated:

$$
\langle q(t+\tau) q(t)\rangle_{D}=2 \pi S_{q} \delta(\tau), \quad\langle f(t+\tau) f(t)\rangle_{D}=2 \pi S_{f} \delta(\tau) .
$$

Here we introduced spectral densities of the output $\left(S_{q}\right)$ and back-action $\left(S_{f}\right)$ noise that are constant in the relevant low frequency range. Besides the infinitely small response time of the detector, eqs. (6) also imply that dynamics of both $q(t)$ and $f(t)$ is essentially classical, since the frequencydependent part of the spectrum that corresponds to zero-point fluctuations gives negligible contribution to the correlators (6).

\section{Back-action dephasing versus acquisition of information}

Qualitatively, measurement dynamics defined by the total Hamiltonian (2) and assumptions (4) and (6) about the detector characteristics, includes two related processes. One is the dephasing and relaxation/excitation of the measured system by the back-action force $f(t)$. This part of the measurement is very similar to the dissipative quantum dynamics of a system 
weakly coupled to a reservoir. The second process is acquisition of information about the state of the measured system reflected in the evolution of the detector output $o(t)$. On the quantitative level, measurement dynamics depends on the Hamiltonian $H_{S}$ of the measured system and the operator structure of the measured observable $x$.

Probably the simplest situation which can be used to illustrate the two qualitative aspects of the measurement dynamics is the case of the stationary system, $H_{S}=0$, with an observable $x$ that has several discrete eigenstates $|j\rangle: x|j\rangle=x_{j}|j\rangle$ with eigenvalues $x_{j}$. Since under the assumption (6) the back-action force $f(t)$ can be treated as a classical random force generated by the detector, one can write the time-evolution equation directly for the density matrix $\rho$ of the measured system. From the Hamiltonian (2), this equation in the basis of $x$-eigenstates is:

$$
\dot{\rho}_{j, j^{\prime}}=\frac{-i}{\hbar}\left(x_{j}-x_{j^{\prime}}\right) f(t) \rho_{j, j^{\prime}} .
$$

Solution of this equation averaged over different realizations of $f(t)$ using eq. (6) gives:

$$
\rho_{j, j^{\prime}}(t)=\rho_{j, j^{\prime}}(0) e^{-\Gamma_{d} t}, \quad \Gamma_{d} \equiv \pi\left(x_{j}-x_{j^{\prime}}\right)^{2} S_{f} / \hbar^{2} .
$$

From this equation one can see that when the system is prepared in the initial state which is a superposition of the eigenstates of $x$, i.e. $\rho_{j, j^{\prime}}$ has nonvanishing off-diagonal elements, interaction with the detector dephases the system and suppresses these off-diagonal elements. The suppression does not happen only if the states $j$ and $j^{\prime}$ are degenerate with respect to $x$ : $x_{j}=x_{j^{\prime}}$, so that the detector does not distinguish them. Equation (7) shows also that the rate $\Gamma$ of such a back-action dephasing is determined by the spectral density of the back-action force $f(t)$.

The dephasing (7) leads to diagonalization of the density matrix in the basis of the measured observable and coincides with the simplest instance of the environment-induced dephasing of an open quantum system. From the measurement perspective, it describes the dynamic side of the "wavefunction collapse", since when the measurement is completed, the measured system should find itself in one of the eigenstates of the observable being measured, i.e. $x$. The density matrix diagonal in the $x$ representation corresponds precisely to such a situation. This interpretation of the back-action dephasing is strengthened by the relation between the dephasing rate $\Gamma$ and the rate of information acquisition - see, e.g., [22]. Indeed, from this perspective, in the example of the preceding paragraph, the detector has to distinguish different eigenstates of the observable $x$ and to provide information in which eigenstate the system finds itself. According to the linear response relation (5), the difference between two eigenvalues 
of $x, x_{j}-x_{j^{\prime}}$, translates into the difference $\delta o=\lambda\left(x_{j}-x_{j^{\prime}}\right)$ of the dc values of the detector output $o$. The characteristic time $\tau_{m}$ on which this difference in $o$ can be distinguished in the presence of the output noise is determined by the low-frequency spectral density $S_{q}$ of this noise. Averaging the $\delta$-correlated noise over the time interval $\Delta t$ leaves characteristic noise amplitude $\Delta o=\left(2 \pi S_{q} / \Delta t\right)^{1 / 2}$. The characteristic time $\tau_{m}$ of acquisition of the information about the dc value of the detector output, and therefore, about the eigenstate of the measured observable, is determined by the condition that the noise amplitude is reduced at least to half the distance $\delta o$ between the signal level. This condition gives

$$
\tau_{m}=8 \pi S_{q} /\left[\lambda\left(x_{j}-x_{j^{\prime}}\right)\right]^{2},
$$

and shows that the relation between the back-action dephasing rate and the "measurement time" $\tau_{m}$ is independent of the eigenvalue difference $x_{j}-x_{j^{\prime}}$, and depends only on the linear-response parameters of the detector:

$$
\tau_{m} \Gamma_{d}=8(\pi / \hbar \lambda)^{2} S_{q} S_{f} .
$$

Now the final step is to show that the linear response theory relates parameters $\lambda, S_{q}, S_{f}$, in such a way that the dephasing rate is fundamentally linked to the measurement time:

$$
\tau_{m} \Gamma_{d} \geq 1 / 2 .
$$

To see this, we start by taking Fourier transform of eq. (4):

$$
-i \frac{\hbar \lambda}{2 \pi}=S_{f q}(\omega)-S_{f q}^{*}(-\omega), \quad S_{f q}(\omega)=\frac{1}{2 \pi} \int d \tau e^{i \omega \tau}\langle f q(\tau)\rangle_{D} .
$$

Expanding expression for the correlator $S_{f q}(\omega)$ in the basis of the energy eigenstates $|\varepsilon\rangle$ of the detector we obtain:

$$
S_{f q}(\omega)=\int d \varepsilon \rho_{D}(\varepsilon) \nu(\varepsilon) \nu(\varepsilon-\hbar \omega)\langle\varepsilon|f| \varepsilon-\hbar \omega\rangle\langle\varepsilon-\hbar \omega|q| \varepsilon\rangle,
$$

where $\nu(\varepsilon)$ is the density of the detector energy states, and since the detector was assumed to be in the stationary state, its density matrix $\rho_{D}(\varepsilon)$ in the energy basis is diagonal.

Making use of the expression (12) and similar expressions for noise spectral densities, we can establish an inequality relating them. To obtain it, we note that eq. (12) can be viewed from a somewhat artificial, but useful perspective as a scalar product of two functions of energy $\varepsilon,\langle\varepsilon-\hbar \omega|q| \varepsilon\rangle$ and $\langle\varepsilon-\hbar \omega|f| \varepsilon\rangle$. Since both the density of states and the probability $\rho_{D}(\varepsilon)$ are non-negative, the scalar product defined by eq. (12) satisfies all the 
usual requirements of the scalar product. Using the standard notation for this product, we can write (12) simply as

$$
S_{f q}(\omega)=<f \mid q>
$$

Spectral densities of the back-action and the output noise can be also expressed in terms of this scalar product. The total spectral densities $S_{q}$, $S_{f}$ are defined as

$$
S_{q}(\omega)=\left(S_{q q}(\omega)+S_{q q}(-\omega)\right) / 2, \quad S_{q q}(\omega)=\frac{1}{2 \pi} \int d \tau e^{i \omega \tau}\langle q q(\tau)\rangle_{D},
$$

with similar equations for $S_{f}(\omega)$. Writing the correlators $S_{q q}(\omega)$ and $S_{f f}(\omega)$ in the same way as $S_{f q}(\omega)$ (12) in the basis of the detector energy eigenstates one sees immediately that they again can be expressed very simply in the language of the introduced scalar product:

$$
S_{q q}(\omega)=<q\left|q>, \quad S_{f f}(\omega)=<f\right| f>.
$$

Schwarz inequality for this scalar product gives, when applied to eqs. (13) and (15):

$$
S_{f f}(\omega) S_{q q}(\omega) \geq\left|S_{f q}(\omega)\right|^{2} .
$$

Finally, combining eq. (11), inequality (16), similar inequality for components of the correlators at frequency $-\omega$, and our assumption that the noise spectral densities $S_{q}(\omega)$ and $S_{f}(\omega)$ are constant at low frequencies, we see that

$$
\lambda=-4 \pi \operatorname{Im} S_{f q} / \hbar
$$

and therefore

$$
|\lambda| \leq \frac{4 \pi}{\hbar}\left[S_{f} S_{q}-\left(\operatorname{Re} S_{f q}\right)^{2}\right]^{1 / 2},
$$

where all spectral densities and the response coefficient are now taken at low frequencies. This inequality is the main result of application of the linear response theory to the measurement problem.

One consequence of the inequality (17) is the proof of the relation (10) between the back-action dephasing rate and the measurement time. It follows directly from eq. (17) that when the detector is "symmetric", i.e. $\operatorname{Re} S_{f q}=0$, so that there are no classical correlations between the backaction noise and the output noise, inequality (17) transforms eq. (9) directly into the (10). (The case of an asymmetric detector is discussed below.) In this derivation of (10), this inequality appears to be the consequence of some relation between the correlators appearing in the linear response theory. From the perspective of the measurement problem it can be interpreted 
in a much broader sense. Inequality (10) shows that in general the dephasing of the measured system by a detector can be arbitrary strong without providing any information on the system. However, acquisition of information about the state of the system creates some minimum dephasing that suppresses coherence between the eigenstates of the measured observable. The fact that such a minimum exists is dictated by the basic principle of quantum mechanics which requires the successful measurement of an observable to localize the system in one of the eigenstates of this observable. If the detector is such that it is causing only this minimum back-action dephasing dictated by quantum mechanics, it is typically referred to as "quantum-limited" or "ideal" detector.

Interplay between information acquisition by the detector and backaction dephasing of the measured system has a simple form of inequality (10) only in the situation of measurement of a static system. When the system Hamiltonian $H_{S}$ is non-vanishing, this interplay is affected by the dynamics of the system and in general manifests itself less directly. One studied example of this $[23,24]$ is the measurement of a two-state system. Coherent quantum oscillations in this system are transformed by measurement into classical oscillations of the detector output. In this case, the trade-off between the back-action dephasing and information acquisition is reflected in the height of the oscillations peak $S_{m}$ in the output spectrum relative to the output noise $S_{q}$. Inequality (10) limits then the peak height: $S_{m} / S_{q} \leq 4$, with eaquality reached by measurement with a symmetric quantum limited detector.

Another system which has been studied in this context is harmonic oscillator $[25,6]$. Similarly to the two-state system, dynamics of the measurement process is reflected in this case in the frequency spectrum of the detector output, which contains the detector output noise and a peak at the oscillator frequency. The trade-off between the detector back-action and information gain becomes relevant if one asks a question what is the minimum contribution of the detector noise to the spectrum. Qualitatively, there are two ways in which the detector noise contributes to the spectrum. The detector output noise gives direct contribution to the spectrum, while the back-action noise contributes to the output spectrum indirectly, by inducing additional oscillations at the detector input that are transformed to the output by the detector response. Minimization of the total noise contribution (reduced to the detector input and normalized to the zero-point spectrum of the oscillator in units of $\hbar / 2$ ) shows that the minimum total noise is given by the "energy sensitivity" $\epsilon$ :

$$
\epsilon=\frac{2 \pi}{|\lambda|}\left[S_{f} S_{q}-\left(\operatorname{Re} S_{q f}\right)^{2}\right]^{1 / 2}
$$


Energy sensitivity has the dimension of action, and relation (17) between the parameters of the linear response theory translates into the following limitation on $\epsilon$ :

$$
\epsilon \geq \hbar / 2 \text {. }
$$

Inequality (19) can be expressed qualitatively as a statement that the detector, when measuring a harmonic oscillator, adds at least half an excitation quantum of this oscillator to the measured signal. This inequality was obtained for the first time in a different physical context of linear amplification of electromagnetic radiation, where this interpretation has direct meaning - see, e.g., the papers on quantum noise in linear amplifies reprinted in [1], also [26] and references therein. The magnitude of the signal in this context is described naturally by the number of photons in a mode and amplification is characterized by the dimensionless photon gain: the ration of these numbers in the output and input modes. Relation between such a linear amplifier and the linear detector considered in this work is established by the fact that in the limit of large gain, the input signal of the amplifier which in general can be quantum (i.e., contain only few photons) is transformed into the output signal which contains number of photons much larger than one and is in this sense classical. This means that the amplifier in this regime acts essentially as a detector, since after amplification of the signal to the classical level, it can be dealt with without any restrictions.

The last point to be made here concerns the case of an asymmetric detector. Such a detector is defined by the nonvanishing classical correlations between the output and back-action noises described by the real part of the spectral density $S_{f q}$. Comparison of eq. (9) for the trade-off between the measurement time and the back-action dephasing, and eq. (19) for the energy sensitivity shows that they behave differently in the case of nonvanishing $\operatorname{Re} S_{f q}$. While the energy sensitivity (19) can reach its quantum limit even in asymmetric detectors with $\operatorname{Re} S_{f q} \neq 0$, the $\tau_{m} \Gamma_{d}$ product (9) in this situation can only be larger than its quantum limit $1 / 2$. The origin of this discrepancy is that in asymmetric detectors the output noise contains some information about the back-action noise which should be utilized to reach the optimum quantum-limited performance of the detector. In the simple treatment that lead to inequality (10), this information is lost, and as a result the dephasing is increased beyond the minimum required by the quantum mechanics. In the case of measurement of the harmonic oscillator, the noise minimization procedure implicitly makes use of this information, since the precise frequency where the noise is minimum depends on the correlation strength $\operatorname{Re} S_{f q}$. In the case of information/dephasing trade-off, the information provided by the correlations contained in non-vanishing $\operatorname{Re} S_{f q}$ can also be used to approach the quantum limit of dephasing. Conceptually 
(and probably also practically) the simplest way to use the information contained in the output noise of the detector to reduce the back-action dephasing is to apply the output noise with an appropriate transfer coefficient to the measured system together with the back-action noise. Although physically this procedure might require a rather complicated set-up that actually applies part of the detector output to the measured system, formally this is achieved by simply redefining the back-action force of the detector:

$$
f \rightarrow f-\left(\operatorname{Re} S_{f q} / S_{q}\right) o \equiv f^{\prime}
$$

It is straightforward to see that the rate $\Gamma_{d}^{\prime}$ of back-action dephasing created by the redefined force $f^{\prime}$ in measurement of a static system can indeed be equal to the quantum minimum of $1 / 2 \tau_{m}$ even for asymmetric detectors with $\operatorname{Re} S_{f q} \neq 0$, if the relation (17) between the linear response properties of this detector is satisfied as equality. The same conclusion can be reached for measurement of the two-state systems [22]. This provides one more example of the fact that many physical characteristics of the measurement process can be understood conveniently as dynamics of information.

\section{Acknowledgements}

The author would like to thank E.V. Sukhorukov for useful discussion of the results and for drawing his attention to Ref. [27]. This work was supported in part by AFOSR and ARDA.

\section{References}

1. Quantum theory and measurement, Ed. by J.A. Wheeler and W.H. Zurek (Princeton Univ. Press, 1983).

2. Quantum optics, experimental gravity, and measurement theory, Ed. by P. Meystre and M.O. Scully (Plenum, NY, 1983).

3. Sixty two years of uncertainty: historical, philosophical, and physical inquiries into the foundations of quantum mechanics, Ed. by A.I. Miller (Plenum, NY, 1990).

4. V.B. Braginsky and F.Ya. Khalili, Quantum measurement, (Cambridge, 1992).

5. M.B. Mensky, Quantum measurement and decoherence: models and phenomenology (Kluwer, Dordrecht, 2000).

6. D.V. Averin, in: "Exploring the quantum/classical frontier: recent advances in macroscopic quantum phenomena", Ed. by J.R. Friedman and S. Han, (Nova Publishes, Hauppauge, NY, 2002), p. 441; cond-mat/0004364.

7. M. Field, C.G. Smith, M. Pepper, D.A. Ritchie, J.E.F. Frost, G.A.C. Jones, and D.G. Hasko, Phys. Rev. Lett. 70, 1311 (1993).

8. M. Kataoka, C.J.B. Ford, G. Faini, D. Mailly, M.Y. Simmons, D.R. Mace, C.-T. Liang, and D. A. Ritchie, Phys. Rev. Lett. 83, 160 (1999).

9. D. Sprinzak, E. Buks, M. Heiblum, and H. Shtrikman, Phys. Rev. Lett. 84, 5820 (2000).

10. S.A. Gurvitz, Phys. Rev. B 56, 15215 (1997).

11. A.N. Korotkov, Phys. Rev. B 60, 5737 (1999).

12. Yu. Makhlin, G. Schön, and A. Shnirman, Phys. Rev. Lett. 85, 4578 (2000). 
13. D.V. Averin, in: "Macroscopic Quantum Coherence and Quantum Computing" Ed. by D.V. Averin, B. Ruggiero, and P. Silvestrini, (Kluwer, 2001), p. 399; condmat/0010052.

14. M.H. Devoret and R.J. Schoelkopf, Nature 406, 1039 (2000).

15. G. Johansson, A. Käck, and G. Wendin, Phys. Rev. Lett. 88, 046802 (2002).

16. A.B. Zorin, Phys. Rev. Lett. 76, 4408 (1996).

17. A.A. Clerk, S.M. Girvin, A.K. Nguyen, and A.D. Stone, Phys. Rev. Lett. 89, 176804 (2002).

18. D.V. Averin, Fortschrit. der Physik 48, 1055 (2000).

19. S. Pilgram and M. Büttiker Phys. Rev. Lett. 89, 200401 (2002).

20. S.-X. Li, Y. Yu, Y. Zhang, W. Qiu, S. Han, and Z. Wang, Phys. Rev. Lett. 89, 098301 (2002).

21. U. Weiss, Quantum dissipative systems, (World Scientific, 1999).

22. A.N. Korotkov, cond-mat/0209629.

23. A.N. Korotkov and D.V. Averin, Phys. Rev. B 64, 165310 (2001).

24. L.N. Bulaevskii, M. Hruska, G. Ortiz, cond-mat/0212049.

25. V.V. Danilov, K.K. Likharev, and A.B. Zorin, IEEE Trans. Magn. 19, 572 (1983).

26. C.M. Caves, Phys. Rev. D 26, 1817 (1982).

27. A.A. Clerk, S.M. Girvin, and A.D. Stone, cond-mat/0211001. 